\title{
ORIGINAL
}

\section{ANTICUERPOS FRENTE A VIRUS WEST NILE Y OTROS VIRUS TRANSMITIDOS POR ARTROPODOS EN LA POBLACION DEL DELTA DEL EBRO}

\author{
Alvaro Lozano (1) y Armindo R Filipe (2) \\ (1) Centro Nacional de Microbiología, Instituto de Salud Carlos III. Ministerio de Sanidad y Consumo. \\ (2) Centro de Estudos de Vectores e Doenças Infecciosas. Instituto Nacional de Saúde.
}

\section{RESUMEN}

Fundamentos: El virus West Nile (VWN) es un Flavivirus que se transmite al hombre a través de distintas especies de mosquitos y produce brotes y casos esporádicon de enfermedad en distintas regiones del Viejo Mundo, incluída la Cuenca Mediterránea. Las zonas húmedas europeas que acogen aves migratorias procedentes de África constituyen áreas de alto riesgo para esta infección, así como para otras infecciones víricas transmitidas por artrópodos.

Métodos: Con objeto de investigar la prevalencia de la infección por el VWN y otros virus de transmisión similar en la población humana del Delta del Ebro, se estudiaron 1037 muestras de suero, obtenidas en 10 localidades de la zona, para presencia de anticuerpos frente a VWN y otros 12 virus transmitidos por artrópodos ( 3 Alfavirus, 8 Flaviviridae y 1 Bunyaviridae) mediante titulación por inhibición de la hemaglutinación (IHA). En algunos casos se estudió la presencia de IgM específica por IHA tras fraccionar el suero por centrifugación en gradientes de sacarosa.

Resultados: En total, se encontró reactividad significativa frente a alguno de los virus probados en 130 casos $(12.5 \%$; $4.1 \%$ frente a Alfavirus, $8.0 \%$ frente a Flaviviridae y $0.4 \%$ frente a Bunyaviridae). El análisis de los títulos de anticuerpos reveló porcentajes significativos de muestras con títulos elevados frente a antígenos de VWN y otros. La distribución de la seroprevalencia fue muy desigual, concentrándose fundamentalmente en 3 localidades del interior del Delta (Ampolla, San Jaime y Montells), donde la prevalencia de anticuerpos frente a Flavivinidae llegó a alcancar el $30 \%$ y se observaron niveles residuales de IgM frente a VWN en algunos sueros.

Conclusiones: Estos resultados y los obtenidos previamente en otras regiones de la Península Ibérica sugieren que el VWN circula en la población humana de las zonas de riesgo y produce brotes epidémicos periódicos. Habida cuenta del alto porcentaje de complicaciones neurológicas observado en los brotes más recientes de infección por $\mathrm{VWN}$ registrados en la cuenca Mediterránea (Bucarest, Argelia), cabe pensar que el VWN juegue algún papel en la etiología de las meningitis y encefalitis víricas que sucedan en la población de las áreas de riesgo del territorio español.

Palabras clave: Virus West Nile. Alfavirus. Flavivirus. Epidemiología. Delta del Ebro.

Correspondencia:

Alvaro Lozano.

Centro Nacional de Microbiología.

Majadahonda

28220 Madrid

Tfno: 5097901; Fax: 5097966
ABSTRACT

\section{Antibodies Against WestNile Virus and other Arthropod-borne Viruses among the Inhabitants of the Ebro Delta}

Background: The West Nile Virus (WNV) is a Flavivirus which is transmitted to man by means of different species of mosquitoes and causes outbreaks and sporadic cases of illness in different regions of the Old World, including the Mediterranean Basin. Europe's wetlands which comprise a stopping-off point for birds migrating from Africa are high-risk areas as regards this infection, as well as other arthropod-borne virus infections.

Methods: For the purpose of researching the prevalence of the WNV infection and other viruses transmitted similarly among the human population of the Ebro Delta, 1037 samples of serum taken in 10 towns in this area were analyzed to detect the presence of WNV antibodies and antibodies of another 12 arthropod-bornc viruses ( 3 Alphaviruscs, 8 Flaviviridac and 1 Bunyaviridae) by means of titration by inhibition of hemagglutination (HAI). In some cases, the presence of HAI-specific IgM was analyzed after breaking down the serum by centrifuging into sucrose gradients.

Results: In all, a significant degree of reaction was found to some of the viruses tested in 130 cases $(12,5 \% ; 4,1 \%$ to Alphavirus: $8 \%$ to Flaviviridae and $0,4 \%$ to Bunyaviridae). The analysis of the antibody titers revealed significant percentages of samples showing high was titers to WNV and other types of antigens. The spread for the antibody prevalence was highly uneven. being focused mainly in 3 localities located in land on the Delta (Ampolla, San Jaime and Montells), where the prevalence of Flaviviridae antibodies totated as high as $30 \%$, with residual levels of WNVrelated IgM having been found in some serum samples.

Conclusions: These results and those obtained previously in other parts of the Iberian Peninsula suggest that the W'NV is moving throughout the human population in the areas where this risk is found to exist and periodically gives rise to epidemic outbreaks. Bearing in mind the high percentage of neurological complications found to exist in the most recent outbreaks of WNV infections recorded in the Mediterranean Basin (Bucharest, Algeria), it can be thought that the WNV plays some role in the factors contributing to viral meningitis and encephalitis which occur within the population of the areas at risk within Spain.

Key Words: West Nile Virus. Alphavirus. Flavivirus; Epidemiology. Ebro Delta. 


\section{INTRODUCCIÓN}

El virus West Nile (VWN) es un miembro de la familia Flaviviridae ampliamente diseminado en Africa y que puede transmitirse al hombre. Las aves constituyen el reservorio principal del VWN y su transmisión a la población humana sucede a través de distintas especies de mosquitos y garrapatas susceptibles a la infección. Aunque los países del nordeste de Africa constituyen las áreas endémicas por excelencia, se han registrado brotes epidémicos de infección por $\mathrm{VWN}$ en puntos tan alejados como el Mediterráneo Oriental', la Camarga francesa $^{2}$ y Sudáfrica ${ }^{3}$ y los estudios de seroprevalencia han revelado situaciones de endemia en la población humana de áreas tan diversas como Madagascar ${ }^{4}$ y Pakistán ${ }^{5,6}$. Recientemente, se ha registrado un amplio brote de infección por VWN en los alrededores de Bucarest?

Habitualmente, la infección por VWN produce un cuadro febril leve y autolimitado, similar a un síndrome gripal, que se sigue a menudo de cuadros prolongados de astenia. Sin embargo, las complicaciones neurológicas pueden ser frecuentes. Así, el brote de Bucarest se registró como un brote de meningitis linfocitaria aguda y produjo una mortalidad cercana al $10 \%$, debida a la aparición de casos de encefalitis ${ }^{7}$. Estos casos graves han sido detectados también como casos esporádicos de encefalitis víri$\mathrm{ca}^{8,9} \mathrm{y}$, más recientemente, en el contexto de un brote de síndrome neurológico agudo en Argelia ${ }^{10}$. Esporádicamente, la infección por VWN puede originar también cuadros de mielitis aguda que recuerdan a la poliomielitis ${ }^{11}$.

Los hábitos migratorios de muchas de las especies de aves capaces de albergar la infección por VWN proporcionan al virus la oportunidad de introducirse en aquéllas áreas que, albergando poblaciones estables de algún vector potencial, son refugio estacional de las aves portadoras. En la Península Ibérica existen varias de ellas y el Delta del Ebro es una de las principales. De hecho, los datos disponibles indican la presencia de mosquitos infectados por VWN en territorio peninsular y de aves y personas con anticuerpos frente a éste y otros virus vehiculados por aves y transmitidos por artrópodos en Portugal y España ${ }^{12}$. Cabe, pués, suponer que el VWN produzca brotes de infección en la población humana de las áreas en riesgo y pueda ser causa de casos esporádicos de infección neurológica aguda en dichas áreas. Al objeto de ampliar los datos en este sentido, se realizó un estudio de seroprevalencia de anticuerpos frente al VWN y otros virus transmitidos por artrópodos entre los habitantes de distintas localidades del Delta del Ebro, provincia de Tarragona.

\section{MATERIAL Y MÉTODOS}

Se estudiaron 1037 muestras de suero de otros tantos residentes en las localidades de Amposta (353 sueros), San Jaime (188), Montells (139), Jesús y María (107), Ampolla (105), San Carlos (56), Camarles (45), La Cava (41), Aldea y Masdenverge (3), recogidas a lo largo del mes de Noviembre de 1980. La muestra incluyó individuos de ambos sexos, con edades comprendidas entre los 6 y los 90 años que se prestaron voluntariamente a la extracción de sangre tras asistir a las charlas impartidas por uno de los autores en lugares de reunión previamente anunciados. Se trató, pués, de un estudio de oportunidad destinado a comprobar la circulación de ciertos virus transmitidos por artrópodos, en el que la única consideración previa fue la selección del área geográfica a estudiar, en base a sus peculiaridades ecológicas. Todos los sueros fueron separados y congelados a $-20^{\circ} \mathrm{C}$ hasta el momento de realizar las pruebas serológicas.

La presencia de anticuerpos frente al VWN (anti-VWN) se investigó por titulación mediante la prueba de inhibición de la hemaglutinación (IHA) ${ }^{13}$, utilizando un antígeno hemaglutinante preparado según el procedimiento de Clarke y Casals ${ }^{13}$ a partir 
de la cepa Ef101. Los sueros se titularon desde la dilución 1/20 por dilución doble seriada hasta la 1/640. En casos seleccionados, se investigó la presencia de anticuerpos anti-VWN de la clase IgM (anti-VWN IgM) realizando la prueba de IHA sobre las fracciones resultantes de la ultracentrifugación de las muestras en gradientes de sacarosa, según un procedimiento descrito y validado previamente $^{14}$.

Paralelamente, se investigó en todas las muestras la presencia de anticuerpos IHA frente a los siguientes virus: Sindbis (VSB) (Eg AR 339); Chikungunya (VCG) (Ross); Semliki Forest (VSF) (aislado original); Encefalitis transmitida por garrapatas (VTBE) (Stillerova); Banzi (VBN) (H336); Ntaya (VNY) (aislado original); Zika (VZK) (aislado original); Wesselsbron (VWS) (N177); Dengue 1 y 2 (VDG1, VDG2) (aislados originales); Fiebre amarilla (VFA) (aislado original); y Bunyamwera (VBY) (aislado original). Los tres primeros pertenecen a los Togaviridae, Alfavirus, los ocho siguientes a los Flaviviridae y el último a los Bunyaviridae. La preparación de los antígenos y las pruebas de IHA se realizaron según los mismos procedimientos aplicados al VWN.

\section{RESULTADOS}

Los resultados globales del estudio se recogen en la tabla 1 , que resume las prevalencias de anticuerpos obtenidas para las distintas familias de virus estudiadas y en cada una de las localidades en las que se obtuvieron muestras. Dado que entre algunos Alfavirus $\mathrm{y}$ algunos Flaviviridae pueden producirse reacciones cruzadas en las pruebas de IHA, sólo se contabilizaron como positivos aquellos sueros que reaccionaron a título igual o superor a 20 , con uno o más virus de la misma familia. En conjunto, un $8 \%$ de las muestras reaccionaron frente a antígenos de Flaviviridae y un $4 \%$ lo hicieron frente a antígenos de Alfavirus, siendo excepcional la reactividad frente a Bunyaviridae (4 muestras). Las prevalencias fueron muy dispares en los distintos núcleos de población estudiados, destacando los altos porcentajes de muestras reactivas frente a antígenos de Flaviviridae encontrados en las localidades de Ampolla (31/105, 29.7\%) Montells

Tabla 1

Reactividad en la prueba de IHA obtenida en 1037 sueros tomados en la región del Delta del Ebro al enfrentarlos a antígenos preparados a partir de cepas de distintos virus pertenecientes al género Alfavirus y a las familias Flaviviridae y Bunyaviridae

\begin{tabular}{|c|c|c|c|c|c|c|c|c|}
\hline \multirow{3}{*}{ Localidad } & \multirow{3}{*}{ Estudiadas } & \multicolumn{6}{|c|}{ Muestras reactivas frente a } & \multirow{3}{*}{$\begin{array}{l}\% \text { total } \\
\text { reactivas }\end{array}$} \\
\hline & & \multicolumn{2}{|c|}{ Alfavirus } & \multicolumn{2}{|c|}{ Flaviviridae } & \multicolumn{2}{|c|}{ Bunyaviridae } & \\
\hline & & + & $\%$ & + & $\%$ & + & $\%$ & \\
\hline Amposta & 353 & 0 & - & 1 & 0,2 & 0 & - & 0,2 \\
\hline Aldea & 1 & 0 & - & 0 & - & 0 & - & - \\
\hline Camarles & 45 & 0 & - & 0 & - & 0 & - & $\ldots$ \\
\hline San Carlos & 56 & 0 & - & 1 & 1,7 & 0 & - & 1,7 \\
\hline Ampolla & 105 & 6 & 5,7 & 31 & 29,7 & 1 & 0,9 & 36,1 \\
\hline Jesús y María & 107 & 9 & 8,4 & 2 & 1,8 & 0 & - & 10,2 \\
\hline La Cava & 41 & 0 & - & 0 & - & 0 & - & - \\
\hline San Jaime & 188 & 24 & 12,7 & 25 & 13,2 & 2 & 1,0 & 27,1 \\
\hline Montells & 139 & 4 & 2,8 & 23 & 16,5 & 1 & 0,7 & 20,1 \\
\hline Masdenverge & 2 & 0 & - & 0 & - & 0 & - & - \\
\hline TOTAL & 1.037 & 43 & 4.1 & 83 & 8,0 & 4 & 0,3 & 12,5 \\
\hline
\end{tabular}


$(23 / 139,16.5 \%)$ y San Jaime $(25 / 188$, $13.2 \%)$ y los correspondientes a Alfavirus en esta última localidad (12.7\%). En contraste y con la excepción de una, las numerosas muestras recogidas en la ciudad de Amposta fueron negativas para todos los antígenos probados.

La tabla 2 resume los títulos de anticuerpos obtenidos en las muestras reactivas para cada uno de los Flaviviridae y los Alfavirus ensayados. Se obtuvieron títulos IHA iguales o superiores a 160 en porcentajes mayores del $25 \%$ del total de muestras reactivas para el Alfavirus VSF $(11 / 32,34.0 \%)$ y los Flaviviridae VWN $(31 / 96,32.3 \%)$ y VFA $(16 / 58,27.5 \%)$.

A la vista de las prevalencias obtenidas, se estudió la presencia de $\operatorname{IgM}$ específica frente a los Flaviviridae en muestras seleccionadas entre las obtenidas en la localidad de Montells. Sólo se obtuvieron algunos resultados positivos, siempre a muy bajo título, para el antígeno de VWN.

\section{DISCUSIÓN}

Los datos sobre la presencia de virus transmitidos por artrópodos en España son aún muy escasos, si bien la situación de estos virus en la Península Ibérica se conoce algo mejor merced a los estudios realizados en Portugal ${ }^{12}$, donde se han conseguido aislamientos de VWN en mosquitos del género Anopheles ${ }^{15} \mathrm{y}$ de los ortomixovirus Dhori y Thogoto ${ }^{16,17}$ y el bunyavirus Palma ${ }^{18}$ en garrapatas. Además, se han detectado anticuerpos en sueros de animales y humanos frente a una larga lista de virus, incluyendo VWN, VSB, VCG y VTBE ${ }^{12}$. En España, se han encontrado prevalencias altas de anticuerpos frente a VWN a alto título en los estudios realizados en Valencia ${ }^{19}$, Galicia ${ }^{20}$ y los alrrededores del Coto de Doñana ${ }^{21}$ en muestras humanas. Los resultados obtenidos en Galicia hacen suponer la existencia de brotes epidémicos de infección por VWN en esa región durante los años sesenta.

En consonancia con estos datos previos, el presente trabajo pone de manifiesto la existencia de una prevalencia alta de anticuerpos anti-VWN en poblaciones humanas concretas de la región del Delta del Ebro, así como de un porcentaje apreciable de casos con altos títulos y de algunas muestras con niveles residuales de IgM específica. Tanto la frecuencia de títulos elevados de anticuerpos anti-VWN como la muy desigual distribución de la prevalencia en las distintas localidades estudiadas hablan a favor de la especificidad de los resultados y sugieren la

Tabla 2

Niveles de reactividad en IHA observados en las muestras positivas en los ensayos de IHA

\begin{tabular}{|c|c|c|c|c|c|c|c|c|c|c|c|c|c|}
\hline \multirow{3}{*}{ Título IHA } & \multicolumn{13}{|c|}{ Muestras reactivas frente a $(\%)$} \\
\hline & \multicolumn{3}{|c|}{ Alfavirus } & \multicolumn{9}{|c|}{ Flaviviridae } & \multirow{2}{*}{$\frac{\text { Bunyaviridae }}{V B Y}$} \\
\hline & $V S B$ & $V C G$ & $V S F$ & $V W N$ & $V T B E$ & $V B N$ & $V N Y$ & $V Z K$ & VWS & $V D G l$ & $V D G 2$ & $V F A$ & \\
\hline $20-80$ & $\begin{array}{c}29 \\
(87,9)\end{array}$ & $\begin{array}{c}42 \\
(100)\end{array}$ & $\begin{array}{c}21 \\
(66,0)\end{array}$ & $\begin{array}{c}65 \\
(67,7)\end{array}$ & $\begin{array}{c}42 \\
(97,7)\end{array}$ & $\begin{array}{c}27 \\
(84,4)\end{array}$ & $\begin{array}{c}15 \\
(100)\end{array}$ & $\begin{array}{c}44 \\
(100)\end{array}$ & $\begin{array}{c}44 \\
(89,8)\end{array}$ & $\begin{array}{c}23 \\
(88,5)\end{array}$ & $\begin{array}{c}11 \\
(91,7)\end{array}$ & $\begin{array}{c}42 \\
(72,5)\end{array}$ & 2 \\
\hline $160-640$ & $\begin{array}{c}4 \\
(12,1)\end{array}$ & 0 & $\begin{array}{c}11 \\
(34,0)\end{array}$ & $\begin{array}{c}31 \\
(32,3)\end{array}$ & $\begin{array}{c}1 \\
(2,3)\end{array}$ & $\begin{array}{c}5 \\
(15,6)\end{array}$ & 0 & 0 & $\begin{array}{c}5 \\
(10,2)\end{array}$ & $\begin{array}{c}3 \\
(11,5)\end{array}$ & $\begin{array}{c}1 \\
(8,3)\end{array}$ & $\begin{array}{c}16 \\
(27,5)\end{array}$ & 2 \\
\hline TOTAL & $\begin{array}{c}33 \\
(100)\end{array}$ & $\begin{array}{c}42 \\
(100)\end{array}$ & $\begin{array}{c}32 \\
(100)\end{array}$ & $\begin{array}{c}96 \\
(100)\end{array}$ & $\begin{array}{c}43 \\
(100)\end{array}$ & $\begin{array}{c}32 \\
(100)\end{array}$ & $\begin{array}{c}15 \\
(100)\end{array}$ & $\begin{array}{c}44 \\
(100)\end{array}$ & $\begin{array}{c}49 \\
(100)\end{array}$ & $\begin{array}{c}26 \\
(100)\end{array}$ & $\begin{array}{c}12 \\
(100)\end{array}$ & $\begin{array}{c}58 \\
(100)\end{array}$ & 4 \\
\hline
\end{tabular}

VSB: Virus Sindbis; VCG: Virus Chikungunya; VSF: Virus Semliki Foresi; VWN: Virus West Nile; VTBE: Virus de la Encefalitis iransmitida por garrapatas: VBN: Virus Banzi: VNY: Virus Ntaya: VZK: Virus Zika: VWS: Virus Wesselsbron; VDG1-VDG2: Virus Dengue 1 y 2; VFA: Virus de la Fiebre amarilla: VBY: Virus Bunyamwera. 
existencia de brotes de infección por VWN en localidades concretas de la región (Ampolla, San Jaime, Montells) y en un tiempo cercano a las fechas de la toma de muestras (últimos años de la década de los 70).

El marcado neurotropismo demostrado por las cepas de VWN responsables de los brotes registrados recientemente en la cuenca mediterránea ${ }^{7.10}$ hace suponer que la circulación del VWN en la región debe traducirse en la aparición de casos de meningitis y encefalitis vírica que queden sin diagnóstico etiológico. Habida cuenta que gran parte de los casos de encefalitis de supuesta etiología vírica que se producen en España quedan sin diagnosticar, aún después de realizar estudios de laboratorio exahustivos ${ }^{22}$, creemos justificado realizar estudios específicos de diagnóstico de infección por VWN en las encefalitis agudas que se produzcan en las zonas de riesgo. Dicho diagnóstico puede realizarse fácilmente en la actualidad mediante amplifícación de secuencias genómicas del VWN por la reacción en cadena de la polimerasa (PCR) en muestras de líquido cefalorraquídeo (LCR) tomadas durante la fase aguda de la enfermedad ${ }^{9}$. Con respecto al resto de los virus incluídos en el estudio, cabe destacar el hallazgo de no pocas muestras reactivas a alto título en IHA frente al Alfavirus Semliki Forest (11 casos de 33 positivos) y el Flavivirus VFA (16 casos de 58 positivos) en el área estudiada. En el estudio realizado en el Coto de Doñana destacó también la frecuente detección de muestras positivas a alto título frente al Alfavirus Chikungunya. Es, por tanto, posible que ambos Alfavirus circulcn cn la población humana de las áreas de riesgo o que las reactividades en IHA respondan a una reacción cruzada con otro Alfavirus prevalente en la Península, antigénicamente relacionado con ellos y aún no identificado. Por su parte, parece poco probable que el VFA circule o haya circulado en la región sin originar casos conocidos de fiebre hemorrágica, por lo que hay que asumir que estas reactividades responden a reacciones cruzadas con cl VWN u otro Flavivirus presente en la zona. El último brote de fiebre amarilla registrado en Europa tuvo lugar en Lisboa a mediados del siglo pasado y originó una elevada mortalidad ${ }^{12}$.

En conclusión, los resultados obtenidos en el Delta del Ebro confirman muchos de los hallazgos de los estudios previos realizados en Valencia, Galicia y Andalucía y, considerando los datos en conjunto, sugieren que el VWN circula significativamente en las poblaciones humanas asentadas en las áreas de riesgo. Así, creemos justificado realizar en dichas áreas estudios destinados a aislar cepas de VWN a partir de los reservorios animales y los vectores potenciales. El impacto que pueda tener el VWN sobre la salud de la población respondería, fundamentalmente, a las complicaciones neurológicas que se asocian a la infección y podría explorarse estudiando los casos de meningitis y encefalitis víricas que se produzcan en las áreas de riesgo mediante el uso de métodos de PCR capaces de detectar la presencia de VWN en el LCR de los pacientes. A la vista de los resultados, no se puede descartar que otros virus transmitidos por artrópodos circulen en ésta y otras zonas de riesgo del Estado Español y tengan algún impacto en Salud Pública.

\section{AGRADECIMIENTOS}

Los autores desean expresar su agradecimiento a D. ${ }^{a}$ M. ${ }^{a}$ Teresa Feliú, de la Dirección Provincial de Salud de Tarragona, al personal de la Cooperativa Médica Arrocera de Amposta y a D. ${ }^{a}$ M. ${ }^{a}$ Angeles Ortega, D. ${ }^{a}$ Margarita Sanz y D. ${ }^{a}$ M. ${ }^{a}$ Rosa Rojas, del Centro Nacional de Microbiología, por su ayuda en la recogida de las muestras y el procesamiento del manuscrito. Así mismo, A D Fernando A Redondo, del Sector de Arbovirus del INSA, Lisboa, por su colaboración técnica en la realización de las pruebas serológicas. Por último, al Dr José M Echevarría por la revisión crítica del manuscrito. 


\section{BIBLIOGRAFÍA}

1. Marberg K, Goldblum N, Sterk V, Jasinka-Klingberg W, Klingberg M. The natural history of West Nile fever: 1, clinical observations during an epidemic in Israel. Am J IIyg 1956; 64:259-269.

2. Panthier R. Epidemiology of the West Nile virus: study of an outbreak in Camargue. Ann Inst Pasteur 1968; 114:518-520.

3. McIntosh B, Jupp P, dos Santos I, Meenehan G. Epidemics of West Nile and Sindbis in South Africat with Culex univittatus Theobald as vector. S Afr J Sci 1976; 295-300.

4. Morvan J, Chin LH, Fontenille D, Rakotoarivonyi I, Coulanges P. Prevalence of antibodies to West Nile virus in youngsters from 5 to 20 years old in Madagascar. Bull Soc Pathol Exot 1991; 84:225234.

5. Sugamata M, Ahmed A, Miura T, Takasu T, Kono R, Ogata T, Kimura-Kuroda J, Yasui K. Seroepidemiological study of infection with West Nile virus in Karachi, Pakistan, in 1983 and 1985. J Med Virol 1988; 26:243-247.

6. Bryan JP, Iqbal M, Ksiazek TG, Ahmed A, Duncan JF, Awan B, Krieg RE, Riaz M, Leduc JW, Nabi S, Qureshi MS, Malik IA, Legters LJ. Prevalence of sand fly fever, West Nile, CrimeanCongo hemorragic fever, and leptospirosis antibodies in Pakistani military personnel. Mil Med 1996; 161:149-153.

7. Anónimo. Viral meningitis. Wkly Epidemiol Rec 1996; 71:296.

8. Flatau F, Kohn D, Dahr O, Vasano N. West Nile fever encephalitis. Isr J Med Sci 1981; 17:1057.

9. Igarashi A, Tanaka M, Morita K, Takasu T, Ahmed A, Ahmed A, Akram DS, Waqar MA. Detection of West Nile and Japanese encephalitis viral genome sequences in cerebrospinal fluid from acute encephalitis cases in Karachi, Pakistan. Microbiol Immunol 1994; 38:827-830.

10. Le Guenno B, Bugermouth A, Azzam T, Bouakaz R. West Nile: a deadly virus? Lancet 1996; $348: 1315$.
11. Gadoth N, Weitzman S, Lehmann EE. Acute anterior myelitis complicating West Nile fever. Arch Neurol 1979; 36:172-173.

12. Filipe AR, Rebelo de Andrade H. Arboviruses in the Iberian Peninsula. Acta Virol 1990; 34:582591.

13. Clarke DH, Casals J. Technique for hemagglutination and hemagglutination inhibition with arthropod-borne viruses. Am J Trop Med Hyg $1958 ; 7: 561-573$.

14. Echevarría MC, Fernández MV, Echevarría JM, Nájera R. Diagnóstico de infecciones primarias y reinfecciones por rubéola en mujeres embarazadas. Rev San Hig Públ 1983; 57:701-713.

15. Filipe AR. Isolation in Portugal of West Nile virus from Anopheles maculipennis mosquitoes. Acta Virol 1972; 16:361.

16. Filipe AR, Casals J. Isolation of Dhori virus from Hyalomma marginatum ticks in Portugal. Intervirology $1979 ; 11: 124-127$.

17. Filipe AR, Calisher CH. Isolation of Thogoto virus from ticks in Portugal. Acta Virol 1984; 28:152155.

18. Filipe AR, Alves MJ, Karabatsos N, Alves de Matos AP, Núncio MS, Baccllar F. Palma virus, a new Bunyaviridae isolated from ticks in Portugal. Intervirology 1994; 37:348-351.

19. Sanchís-Bayarri V. Contribución al estudio de la serología de las infecciones por Arbovirus. Hospital General 1974; 14:417-424.

20. Garea MT, Filipe AR. Antibodies to arboviruses in northwestern Spain. Am J Trop Med Hyg 1977; 26:792-797.

21. Lozano A. Arboviruses in Spain. En "Arboviruses in the Mediterranean countries". J.VesenjakHirjan ed.. Gustav Fisher Verlag. Stuttgart, 1980.

22. Echevarría JM, Casas I, de Ory F, Tenorio A, Echevarría C, Lozano A. Diagnóstico de laboratorio en casos de encefalitis aguda y subaguda de posible etiología vírica. Neurología 1997; 12:381383. 